\title{
Chemical behavior of the dwarf irregular galaxy NGC6822. Its PN and $\mathrm{HII}$ region abundances ${ }^{\star} \star \star$
}

\author{
L. Hernández-Martínez, M. Peña, L. Carigi, and J. García-Rojas
}

\begin{abstract}
Instituto de Astronomía, Universidad Nacional Autónoma de México, Apdo. Postal 70264, Méx. D. F., 04510 Mexico
e-mail: [lhernand;miriam;carigi;jgarcia]@astroscu.unam.mx
\end{abstract}

Received 13 May 2009 / Accepted 13 June 2009

\section{ABSTRACT}

\begin{abstract}
Aims. We aim to derive the chemical behavior of a significant sample of PNe and HII regions in the irregular galaxy NGC 6822. The selected objects are distributed in different zones of the galaxy. Our purpose is to obtain the chemical abundances of the present interstellar medium (ISM), represented by $\mathrm{H}$ II regions, and the corresponding values at the time of formation of PNe. With these data the chemical homogeneity of NGC 6822 were tested and the abundance pattern given by H II regions and PNe used as an observational constraint for computing chemical evolution models to infer the chemical history of NGC 6822.

Methods. Due to the faintness of PNe and H II regions in NGC 6822, to gather spectroscopic data with large telescopes is necessary. We obtained a well suited sample of spectra by employing VLT-FORS 2 and Gemini-GMOS spectrographs. Ionic and total abundances were calculated for the objects where electron temperatures could determined through the detection of [O III] $\lambda 4363$ or/and [N II] $\lambda 5755$ lines. A "simple" chemical evolution model was developed and the observed data were used to compute a model for NGC 6822 in order to infer a preliminary chemical history in this galaxy.

Results. Confident determinations of $\mathrm{He}, \mathrm{O}, \mathrm{N}, \mathrm{Ne}, \mathrm{S}$ and $\mathrm{Ar}$ abundances were derived for a sample of $11 \mathrm{PNe}$ and one $\mathrm{H}$ II region. We confirm that the present ISM is chemically homogeneous, at least in the central $2 \mathrm{kpc}$ of the galaxy, showing a value $12+\log \mathrm{O} / \mathrm{H}=8.06 \pm 0.04$. From the abundance pattern of PNe, we identified two populations: a group of young PNe with abundances similar to $\mathrm{H}$ II regions and a group of older objects with abundances a factor of two lower. A pair of extreme Type I PNe were found. No third dredge-up $\mathrm{O}$ enrichement was detected in PNe of this galaxy. The abundance determinations allow us to discuss the chemical behavior of the present and past ISM in NGC 6822. Our preliminary chemical evolution model predicts that an important gas-mass loss occurred during the first $5.3 \mathrm{Gyr}$, that no star higher than $40 M_{\odot}$ was formed, and that $1 \%$ of all $3-15 M_{\odot}$ stars became binary system progenitors of SNIa.
\end{abstract}

Key words. ISM: abundances - HII regions - planetary nebulae: general - galaxies: dwarf - galaxy: abundances - galaxies: evolution

\section{Introduction}

Dwarf irregular galaxies are the "simplest" galactic systems known. They are considered one of the building blocks of the Universe and apparently are dominated by dark matter (Carigi et al. 1999). Two of their main characteristics are their low mass and low metallicity. They are gas-rich galaxies and thought to be chemically homogeneous. Even when they are simple in appearance, they are dominated by star forming complexes and OB associations with typical diameters of 200-300 pc (Fisher \& Tully 1979; Hodge et al. 1991) which are immersed in an older and more extended populations.

NGC 6822 (DDO 209, IC 4895) is one of the closest gas-rich dwarfs in the Local Group. The galaxy appears very extended on the sky due to its small distance from the Milky Way; its optical apparent dimensions are about $15.5^{\prime} \times 13.5^{\prime}$, and it is at a distance

\footnotetext{
* Based on observations collected at the European Southern Observatory, VLT, Paranal, Chile, program ID 077.B-0430.

$\star \star$ Based on observations obtained at the Gemini Observatory (program ID G-2005B-56), which is operated by AURA, Inc. under a cooperative agreement with the NSF on behalf of the Gemini partnership: the National Science Foundation (USA), the Science and Technology Facilities Council (United Kingdom), the National Research Council (Canada), CONICYT (Chile), the Australian Research Council (Australia), Ministério da Ciencia e Tecnologia (Brazil) and SECYT (Argentina).
}

modulus of $23.31 \pm 0.02$ as reported by Gieren et al. (2006). It is moving at $V_{\text {hel }}=-54 \pm 6 \mathrm{~km} \mathrm{~s}^{-1}$ and presents an optical radius of $2.9 \mathrm{kpc} \times 2.9 \mathrm{kpc}$ (Mateo 1998). A huge rotating H I disk of about $6 \mathrm{kpc} \times 14 \mathrm{kpc}$ size at P.A. $\sim 110^{\circ}$ is a well known feature in this galaxy (de Blok \& Walter 2000 and references therein). Its young stellar content extends in zones with radii over $5 \mathrm{kpc}$ from the center (de Blok \& Walter 2006) and it also shows an even larger distribution of faint $\mathrm{C}$ stars forming a spheroid whose long axis lies almost perpendicular to the H I disk (e.g., Demers et al. 2006). NGC 6822 was believed to be isolated, but recent studies argue for the presence of a "North West" companion (de Blok \& Walter 2000, 2006). This galaxy has a total luminosity of $M_{\mathrm{B}}=$ -15.8 (Hodge et al. 1991) and a total H I mass of $1.3 \times 10^{8} M_{\odot}$ (de Blok \& Walter 2006).

Chemically NGC 6822 is a metal-poor galaxy, with an interstellar medium (ISM) abundance of about $0.2 Z_{\odot}$ (Richer \& McCall 2007). Its seems to be chemically homogeneous, as expected for dwarf irregulars. Lee et al. (2006) tested the existence of a possible radial gradient with no conclusive results. So far, a few bright H II regions have been studied by different authors. From collisionally excited lines, Hidalgo-Gámez et al. (2001) reported $12+\log (\mathrm{O} / \mathrm{H})=8.10$ and 8.12 for the regions Hubble V (H V) and Hubble X (HX); Peimbert et al. (2005) re-analyzed the same regions, deriving similar values from collisionally excited lines, while from recombination lines they obtained $12+\log (\mathrm{O} / \mathrm{H})=8.42$. The latter value is more in 
agreement with the values reported for A type supergiants (Venn et al. 2000).

Regarding planetary nebulae (PNe), due to their faintness only a few spectra have been analyzed in the past (Dufour \& Talent 1980; Richer \& McCall 1995). Richer \& McCall (2007) obtained spectroscopic data of a sample of seven PNe. They reported that PNe in NGC 6822 have oxygen and neon abundances very similar to those in the $\mathrm{H}$ II regions.

It has been generally accepted that $\alpha$-elements like oxygen and neon are not affected significantly by the nucleosynthesis processes that occurred during the evolution of low-intermediate mass stars (LIMS), progenitors of PNe, which on the other hand do modify $\mathrm{He}, \mathrm{N}$ and $\mathrm{C}$. Therefore, $\mathrm{O}$ and $\mathrm{Ne}$ of PNe would reflect the metallicity of the ISM from which the parent stars were formed and their abundances were usually taken as representative of the ISM in that epoch. Recently, some observational evidence and theoretical models have shown that this is questionable; $\mathrm{O}$ and $\mathrm{Ne}$ original abundances in $\mathrm{PNe}$ can be perturbed by stellar nucleosynthesis in at least two ways. In more massive progenitors, O can be depleted through the ON-cycle (e.g., Peimbert 1985; Henry 1990; Costa et al. 2000). On the other hand, low metallicity PN progenitors can dredge up freshly synthesized $\mathrm{O}$ (and possibly also $\mathrm{Ne}$, besides $\mathrm{C}$ ) to their surfaces, in the third dredge-up episode (Marigo 2001; Herwig 2004; Leisy \& Dennefeld 2006; Wang \& Liu 2008), although this apparently happens significantly only at very low metallicity. For instance, in some very metal poor galaxies it has been found that oxygen abundances of PNe (and apparently also $\mathrm{Ne}$ ) are higher than the average value of $\mathrm{H}$ II regions. Such is the case in the only PN known in Sextans A (Kniazev et al. 2005; Magrini et al. 2005), in the 8 PNe studied in NGC 3109 by Peña et al. (2007), in a large fraction of $\mathrm{PNe}$ in the $\mathrm{SMC}$ and in a small but significant fraction in the LMC (Leisy \& Dennefeld 2006). Thus, neither O nor Ne can be considered safe for testing the original chemical composition of progenitor stars.

Our main goal in this work is to analyze the chemical abundances of a larger sample of PNe in NGC 6822 and to compare them with the present ISM abundances. In this galaxy there are 26 PN candidates detected (Hernández-Martínez \& Peña 2009, hereafter Paper I) from which we selected a sample for spectroscopic analysis. Based on well measured electron temperatures $\mathrm{T}(\mathrm{O}$ III) we determine the chemical abundances of $11 \mathrm{PNe}$ and one $\mathrm{H}$ II region and analyze their abundance pattern. These data are used to compute one "simple" chemical evolution model for NGC 6822 in order to infer a preliminary chemical history in this galaxy. In a future paper (Hernández-Martínez et al. 2009, in preparation), we will present a more detailed grid of models and a deeper discussion on this subject.

The paper is organized as follows: In Sect. 2 we present the observations and data reduction. Analysis of the spectra and plasma diagnostic are presented in Sect. 3. The behavior of chemical abundances is discussed in Sect. 4 and in Sect. 5 the history of chemical enrichment in NGC 6822 is analyzed by means of a model of chemical evolution. Our conclusions are presented in Sect. 6.

\section{Observations and data reduction}

To accurately determine nebular abundances, data in a large wavelength range, at resolutions large enough to easily separate important diagnostic lines such [O III] $\lambda 4363$ from $\mathrm{H} \gamma$ and the components of the doublet [S II] $\lambda \lambda 6717,6731$, are required. Also exposure times should be long enough to obtain the faint diagnostic lines (usually hundreds of times fainter that $\mathrm{H} \beta$ ) with a good signal-to-noise ratio. In particular, it is crucial to measure the temperature sensitive [O III] 4363/5007 ratio.

With this purpose, we obtained two set of observations with large aperture telescopes by employing multi-object spectrographs (MOS) in order to simultaneously observe a large number of PNe and compact H II regions. First, a service-mode run with the Gemini South telescope and the GMOS spectrograph (2 fields centered at 19:44:56-14:42:58 and 19:44:59-14:48:08, each with size $5.5 \times 5.5$ arcmin, were observed) was performed in 2005 which allowed us to gather high-quality data for some PNe and a few HII regions (program ID GS-2005B-Q-56). A second run with the ESO Very Large Telescope UT1 (Antu) and the FORS2 spectrograph, in MXU mode (covering one field centered at 19:44:56.7-14:47:57.0,6.8 66.8 arcmin size) and also in long-slit mode, was performed on 2006 August 20 and 21. Both GMOS and FORS2-MXU employ masks constructed from previously acquired images (pre-imaging). The log of observations is presented in Table 1 . The objects for spectroscopy were selected from the lists of PN candidates and $\mathrm{H}$ II regions reported by Leisy et al. (2005) and in Paper I. The objects are designed as in Paper I, where the reader can find the cross-correlation with other names in the literature.

Gemini South GMOS pre-imaging was acquired in August 2005, and used to select objects for spectroscopy. Spectroscopic observations were performed through gratings B600 and R600 with a spectral resolution of 0.45 and $0.47 \AA /$ pix respectively. The covered wavelength range depends on the position of the object in the field of view but in general the range from $4000 \AA$ to $7500 \AA$ was obtained. The slit width was $1^{\prime \prime}$ for all the objects, but of varying lengths to accommodate a larger number of objects. In this case, the exposure times were not long enough for the fainter objects whose faint lines were underexposed. Therefore, from these observations, we report only two $\mathrm{PNe}$ and three faint $\mathrm{H}$ II regions which have good enough signalto-noise ratios for plasma analysis. The standard stars LTT 9239 and H 600 were used for flux calibration.

For the spectroscopic VLT-FORS2 run, the grisms 600B and 600RI were used. Again, the spectral range coverage depends on the position of the object, but in general it goes from about $3700 \AA$ to $7500 \AA$ with a spectral resolution of 0.7 to $1.2 \AA /$ pix. The slit width was $1^{\prime \prime}$ for all the objects. The standard stars EG 274, LDS 749B and BMP 16274 were observed through a slit of $5^{\prime \prime}$ width for flux calibration. During the spectroscopic run, the sky was clear and the seeing conditions varied from $0.7^{\prime \prime}$ to $0.9^{\prime \prime}$.

In both set of observations, the spectral resolution is good enough to safely separate lines such as [O III] $\lambda 4363$ from $\mathrm{H} \gamma$ and the doublet [S II] $\lambda \lambda 6717,6731$, which are important for plasma diagnostics.

\subsection{Data reduction, spectral calibrations, and reddening correction}

Normal data reduction was performed by using $\operatorname{IRAF}^{1}$ reduction packages. Raw frames were bias subtracted and flat-fielded. Wavelength calibration was performed with a $\mathrm{He}-\mathrm{Hg}-\mathrm{Cd}$ lamp for FORS2 data and a $\mathrm{Cu}$-Ar lamp for GMOS data. Flux calibration was performed via the standard stars mentioned above.

\footnotetext{
1 IRAF is distributed by the National Optical Astronomy Observatories, which is operated by the Association of Universities for Research in Astronomy, Inc., under contract to the National Science Foundation.
} 
Table 1. Log of observations.

\begin{tabular}{lrrr}
\hline \hline Objects $^{1}$ & Spectrum mode & Grism (exp.time (s) $\times \#$ exposures $)$ \\
\hline VLT FORS2 & Long slit & 600B $(1500 \times 3)$ & 600RI $(1500 \times 2)$ \\
PN 4, PN 6 & MXU & 600B $(1800 \times 3)$ & 600 RI $(1500 \times 3)$ \\
PN 10, PN 12, PN 14, PN 16, PN 18 & & & \\
PN 19, PN 21, H II 15, H II 9 & GMOS & B600 $(1100 \times 4)$ & R600 $(900 \times 4)$ \\
\hline $\begin{array}{c}\text { Gemini south } \\
\text { N: PN 5, PN 7, H II 2, H II 4, H II 5 }\end{array}$ & GM
\end{tabular}

${ }^{1}$ Names from the list by Hernández-Martínez \& Peña (2009); ${ }^{2}$ R600 was used with the filter GG455_G0329.

Line fluxes were measured with the IRAF routine "onedspec.splot", by applying a Gaussian profile fit. For the case of VLT-FORS2 observations, the red and blue spectra of each object were scaled to a common level using lines detected in both spectral ranges. The logarithmic reddening correction at $\mathrm{H} \beta$ was obtained from the Balmer decrement. Spectral lines were dereddened with the Seaton extinction law (Seaton 1979). The dereddened fluxes are presented in Table 2 where the reddening law is also listed. For the objects observed with Gemini-GMOS (PN 5, PN 7 and HII 4) such a procedure cannot be performed because the blue and red spectra do not overlap; they were observed on different days and under different conditions, and the flux calibration is not confident. Thus the Balmer line ratios, $\mathrm{H} \alpha / \mathrm{H} \beta$ and $\mathrm{H} \gamma / \mathrm{H} \beta$, indicate very different reddening corrections. As it is very important for abundance determination to use the [O III] $\lambda 4363$ and other diagnostic line intensities as reliable as possible, we used observed $\mathrm{H} \alpha / \mathrm{H} \beta$ and $\mathrm{H} \gamma / \mathrm{H} \beta$ ratios and we calculated the additional corrections to bring them to the theoretical recombination values. Such a correction was then applied to the observed flux at other wavelengths.

It is interesting to notice that the objects located in the center, near or on the "galactic bar", such as H II 15, Н II 9, PN 10 present higher reddening - on average $E(V-B) \sim$ 0.54 - than the objects located in the periphery, such as (PN 14,PN 16,PN 18,PN 19) which on average show $E(B-V) \sim$ 0.25 . This is in agreement with previous results indicating a reddening varying from $E(B-V)=0.24$ at the border of the galaxy to $E(B-V)=0.45$ in the center (Massey et al. 1995).

Our spectra of PNe and one $\mathrm{HII}$ region are shown in Fig. 1.

\section{Spectral analysis}

\subsection{Plasma diagnostic}

The wide spectral coverage allowed us to determine electron temperature and densities from different diagnostic sensitive ratios; electron temperatures were determined from the [O III] $\lambda \lambda 4363 / 5007$ and [N II] $\lambda \lambda 5755 / 6583$ ratios, when available. Only a few objects show the auroral [N II] $\lambda 5755$ line intense enough to be measured, but most of the high-excitation nebulae present [O III] $\lambda 4363$ with a good signal-to-noise ratio. Nebular densities were obtained through the [S II] $\lambda \lambda 6717 / 6731$ line ratios and occasionally from [Ar IV] $\lambda \lambda 4711 / 4740$, although the latter is very uncertain due to the faintness of the lines. For these calculations, the IRAF routine "temden" of the package "nebular" (Shaw \& Dufour 1995) was used. The results are presented in Table 3.

\subsection{Ionic and total abundances}

To derive ionic abundances, data were treated as homogeneously as possible. Ionic abundances of heavy elements, relative to $\mathrm{H}^{+}$, were computed from the line intensities of Table 2 and the physical conditions described in the above section. In all cases, we adopted a one-electron temperature scheme for all the ions by using T(O III) (the most confident electron temperature) as representative of the whole nebula. Only in the cases where T(O III) could not be determined did we use T(N II) if available. The electron density derived from the [S II] sensitive ratio was used in all cases. The IRAF task "ionic", from the package "nebular", was used.

To determine $\mathrm{He}^{+} / \mathrm{H}^{+}$, we used three observed lines of $\mathrm{He}$ I at $\lambda \lambda 4471,5876$ and 6678 , weighted by 1:3:1. Case B He I emissivities were taken from the collision-less (low-density limit) calculations by Bauman et al. (2005). We used a J-resolved code available on-line ${ }^{2}$ for these calculations. The collisional to recombination contribution was estimated from Kingdon \& Ferland (1995), using the interpolation formula provided by Porter et al. (2007). The effective recombination coefficients for $\mathrm{H}^{+}$were taken from Storey \& Hummer (1995). The $\mathrm{He}^{++}$ abundance was determined from the He II $\lambda 4686$ recombination line, using the recombination coefficients by Storey \& Hummer (1995).

The derived ionic abundances and their uncertainties are listed in Table 3. The uncertainties for heavy element ionic abundances are mainly due to uncertainties in the electron temperature, while for recombination lines, it is due to line intensities errors.

To derive total abundances, the unseen ions should be taken into account. For this we have adopted the ionization correction factors (icf) proposed by Kingsburgh \& Barlow (1994). Then total abundances correspond to the addition of observed ionic abundances multiplied by the corresponding icf. The final results are presented in Table 4, where we have separated PNe from H II regions and we have included some results for well determined $\mathrm{H}$ II regions from the literature.

\subsection{Supernova remnants?}

A few faint nebulae were marked by Leisy et al. (2005) as possible supernova remnants (SNR) due to their intense [S II] lines. Those were (in parenthesis we give Leisy et al. nomenclature) H II 2 (H II 21), H II 4 (H II 20), H II 6 (H II 23) and H II 9 (H II 24). We obtained spectra of three of them (H II 2, 4, and 9), finding they are very low excitation $\mathrm{H}$ II regions, with very faint or no [O III] $\lambda 5007$ emission and intense low excitation lines. The ionizing stars should be later than B0 stars. All these objects are of low density. We estimated the chemical composition for $\mathrm{H}$ II 9 andH II 4 by assuming electron temperatures of $12000 \mathrm{~K}$ and we found a normal chemical composition.

$\overline{2}$ Available at http://www.pa.uKy.edu/ rporter/j-resolved/ 


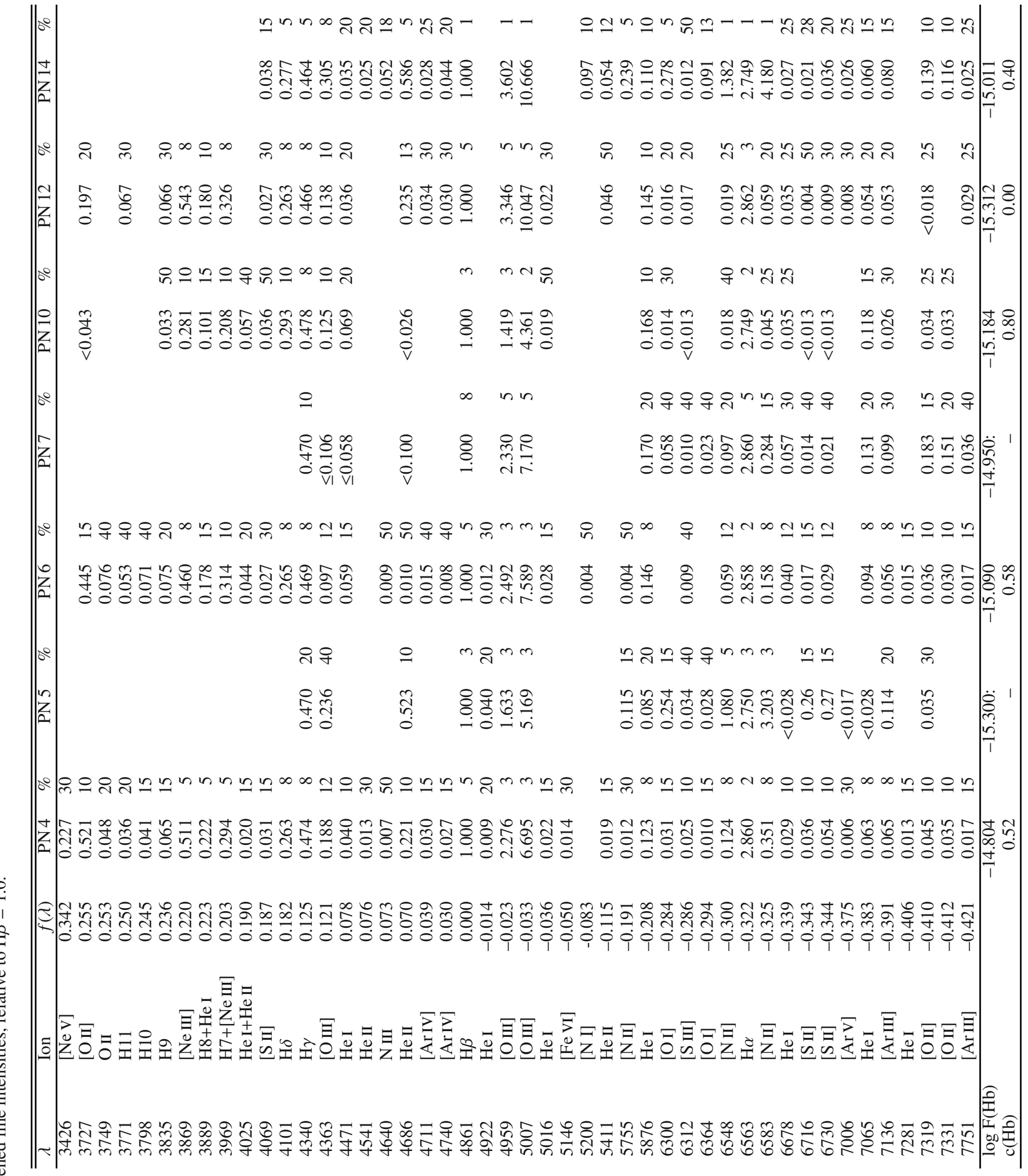




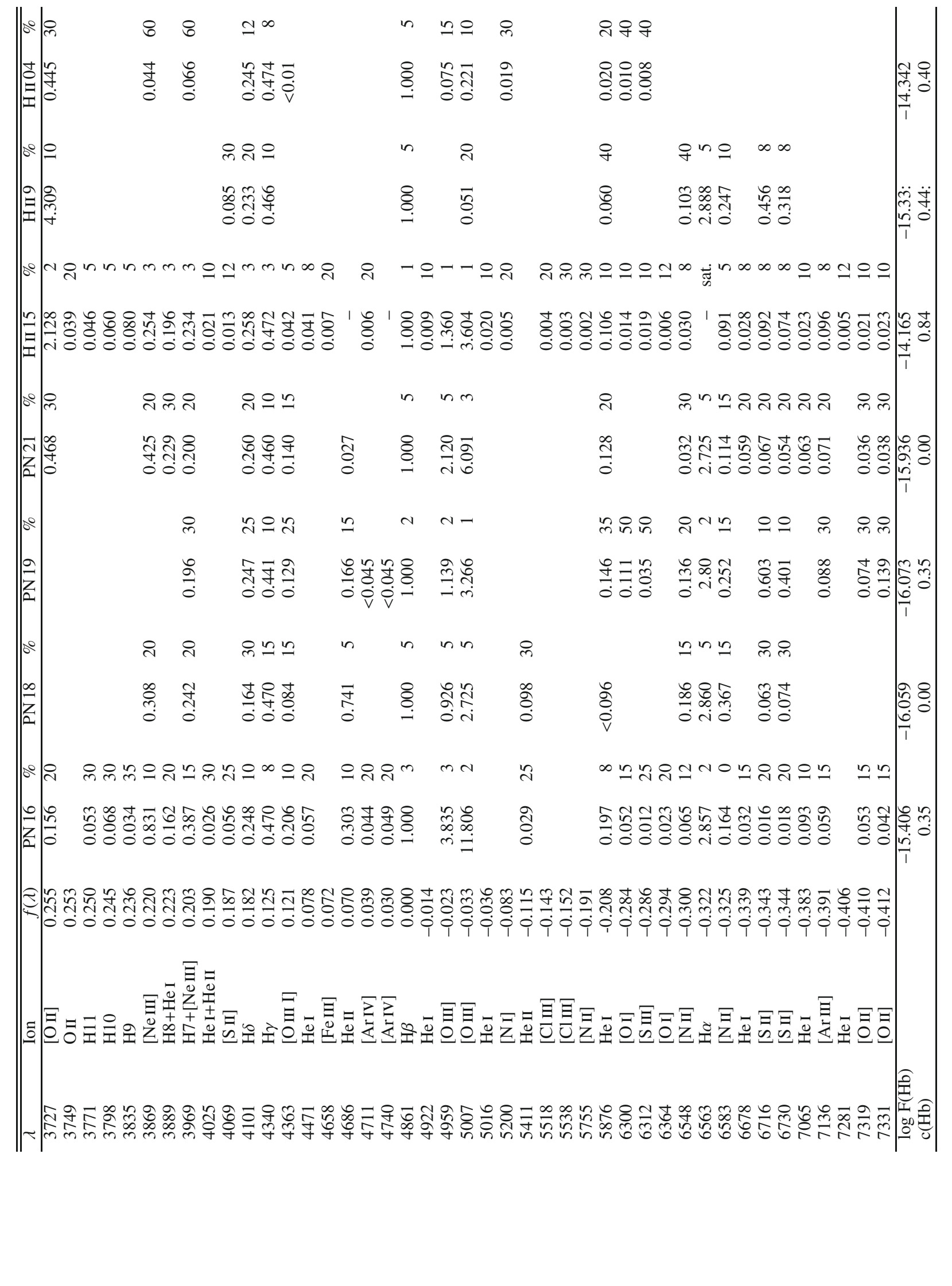




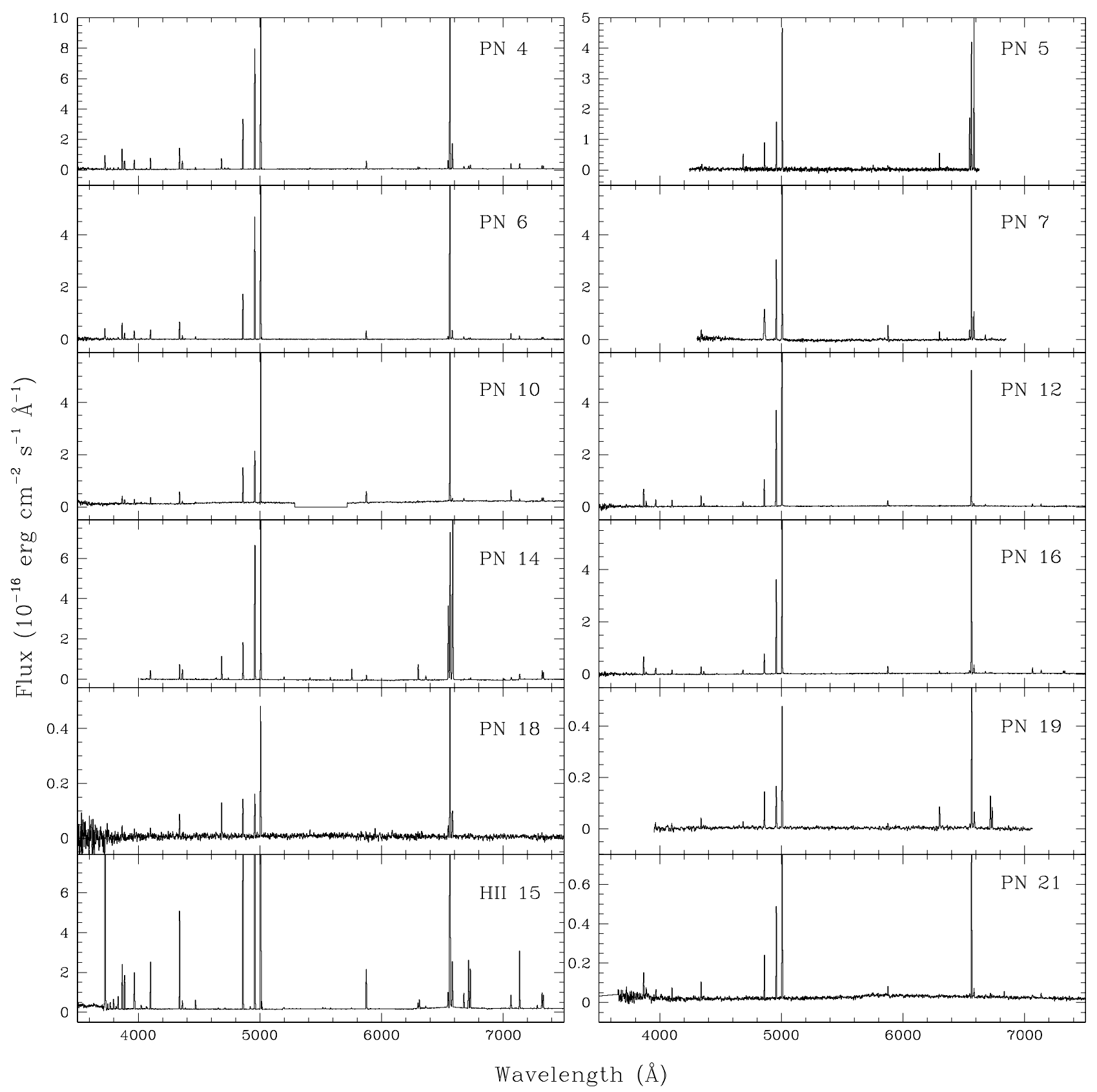

Fig. 1. Spectra of the PNe and the bright H II 15. Blue and red spectra have been combined.

\section{Chemical abundances}

\subsection{Comparison with previous determinations}

From our data we have determined $\mathrm{He}, \mathrm{N}, \mathrm{O}, \mathrm{Ne}, \mathrm{S}$ and $\mathrm{Ar}$ for most of the observed objects. Our sample contains $4 \mathrm{PNe}$ in common with Richer \& McCall (2007, hereafter RMcC), which are PN 10 (their PN 19), PN 21 (their PN 12), PN 14 (their S 33) and PN 16 (their S 16). We observed and re-observed as many PNe as possible in order to secure homogeneous and more precise data. Comparing our derived physical conditions and chemical abundances with those by $\mathrm{RMcC}$, we found that:

- the physical conditions, particularly the electron temperature, are very similar. Densities are similar within uncertainties;

- our He abundances are equal within uncertainties for all the objects but $\mathrm{PN} 16$, where they found an $\mathrm{He} / \mathrm{H}$ ratio $40 \%$ lower. This may be due to their much higher reddening that largely affects the He I $\lambda 5876$ intensity;

- the $\mathrm{O}$ abundances are very similar. Their $\mathrm{O} / \mathrm{H}$ values differ from ours only by 0.06 dex at the most. But other elements are not so consistent. Their N/O for PN 14 is too large (7.94) and for PN 21 (0.03) is too low, compared to our values. These differences are mainly due to N/O abundance ratios based on the $\mathrm{N}^{+} / \mathrm{O}^{+}$ionic abundance ratios, both ions being very under-abundant in high excitation PNe. In particular, the $\mathrm{O}^{+}$abundance is very uncertain due to uncertainties in the [O II] $\lambda 3727$ line which in general is weak; it depends strongly on the adopted temperature and density, and it is very affected by errors in the adopted reddening. For Ne/O ratios, the differences are small except for one object: PN 21, for which we obtain $\mathrm{Ne} / \mathrm{O}=0.18$ and $\mathrm{RMcC}$ derived 0.363 with a large error. We consider our determination to be more confident due to the higher $\mathrm{S} / \mathrm{N}$ of our [Ne III] lines. 
Table 3. Physical conditions and ionic abundances in PNe and $\mathrm{H}$ II regions.

\begin{tabular}{|c|c|c|c|c|c|c|c|}
\hline & PN 4 & PN $5^{1,2}$ & PN 6 & $\mathrm{PN} 7^{1}$ & PN 10 & PN 12 & PN 14 \\
\hline $\mathrm{T}(\mathrm{O}$ III $)(\mathrm{K})$ & $17870 \pm 980$ & $<23000$ & $12590 \pm 1500$ & $<13400$ & $17000 \pm 1000$ & $13000 \pm 1000$ & $18070 \pm 760$ \\
\hline $\mathrm{T}(\mathrm{N}$ II $)(\mathrm{K})$ & $15830 \pm 2300$ & $16100 \pm 1700$ & $12082 \pm 1800$ & & & & $17550 \pm 1000$ \\
\hline $\mathrm{N}(\mathrm{S}$ II $)\left(\mathrm{cm}^{-3}\right)$ & $2980 \pm 360$ & $880 \pm 200$ & $3910 \pm 480$ & $4000 \pm 2000$ & 1000 (adopt) & $2000 \pm 1000$ & $5150 \pm 500$ \\
\hline $\mathrm{N}(\mathrm{Ar} \mathrm{IV})\left(\mathrm{cm}^{-3}\right)$ & $3240 \pm 1000$ & & 1000: & & & $2700 \pm 1000$ & $15700 \pm 3500$ \\
\hline $\mathrm{He}^{+}$ & $7.05 \pm 0.40 \mathrm{E}-2$ & $5.2 \pm 1.1 \mathrm{E}-2$ & $9.52 \pm 0.50 \mathrm{E}-2$ & $1.07 \pm 0.25 \mathrm{E}-1$ & $1.11 \pm 0.08 \mathrm{E}-1$ & $9.34 \pm 0.6 \mathrm{E}-2$ & $5.69 \pm 0.33 \mathrm{E}-2$ \\
\hline $\mathrm{He}^{++}(4686)$ & $0 \pm 0.18 \mathrm{E}-2$ & $4.20 \pm 0.40 \mathrm{E}-2$ & $8.1 \pm 4.0$ E-4 & $0.00 \mathrm{E}+00$ & $0.00 \mathrm{E}+00$ & 0.30 E-2 & $5.20 \pm 0.10 \mathrm{E}-2$ \\
\hline $\mathrm{O}^{+}\left(3^{-}\right.$ & $1 \pm 0.60$ E-6 & $7.7 \pm 2.3$ E $-6^{3}$ & $1.17 \pm 0.26 \mathrm{E}-5$ & $5.50 \pm 1.50 \mathrm{E}-5^{3}$ & $5.85 \pm 1.17 \mathrm{E}-6^{3}$ & $3.16 \pm 1.05$ E- 6 & $1.15 \pm 0.40 \mathrm{E}-5^{3}$ \\
\hline $\mathrm{O}^{++}($ & $9 \pm 0.53 \mathrm{E}-5$ & $4.6 \pm 1.0 \mathrm{E}-5$ & $1.30 \pm 0.20 \mathrm{E}-4$ & $1.02 \pm 0.20 \mathrm{E}-4$ & $3.43 \pm 0.52 \mathrm{E}-5$ & $1.56 \pm 0.40 \mathrm{E}-4$ & $7.32 \pm 0.60 \mathrm{E}-5$ \\
\hline $\mathrm{N}^{+}(6$ & $3 \pm 0.20 \mathrm{E}-6$ & $2.20 \pm 0.50 \mathrm{E}-5$ & $1.87 \pm 0.22 \mathrm{E}-6$ & $\pm 1.0 \mathrm{E}-6$ & $2.54 \pm 0.27 \mathrm{E}-7$ & $6.5 \pm 1.0 \mathrm{E}-7$ & $2.44 \pm 0.30 \mathrm{E}-5$ \\
\hline $\mathrm{Ne}^{+}$ & $0 \pm 1.00 \mathrm{E}-6$ & - & $2.18 \pm 0.35 \mathrm{E}-5$ & - & $5.42 \pm 0.74$ E- 6 & $2.36 \pm 0.66 \mathrm{E}-5$ & $5.80 \pm 0.50 \mathrm{E}-6^{5}$ \\
\hline $\mathrm{Ne}^{+4}$ & $2 \pm 0.45 \mathrm{E}-8$ & - & $0.00 \mathrm{E}+00$ & - & $0.00 \mathrm{E}+00$ & - & - \\
\hline$S^{+}\left(6^{\prime}\right.$ & $3 \pm 0.10 \mathrm{E}-7$ & $5.5 \pm 1.3 \mathrm{E}-7$ & $1.14 \pm 0.17 \mathrm{E}-7$ & $7.8 \pm 2.0 \mathrm{E}-8$ & & 0.60 E-8 & $7.93 \pm 1.00 \mathrm{E}-8$ \\
\hline $\mathrm{S}^{++}(6$ & $3 \pm 1.20 \mathrm{E}-7$ & $2.3 \pm 1.0 \mathrm{E}-6$ & $8.45 \pm 1.65 \mathrm{E}-7$ & $7.75 \pm 2.00 \mathrm{E}-7$ & & $1.54 \pm 0.50 \mathrm{E}-6$ & $4.1 \pm 2.0 \mathrm{E}-7$ \\
\hline $\operatorname{Ar}^{++}(7135)$ & $96 \pm 0.17$ E-7 & $4.10 \pm 0.80 \mathrm{E}-7$ & $3.12 \pm 0.32 \mathrm{E}-7$ & $4.9 \pm 2.0 \mathrm{E}-7$ & $7.9 \pm 1.4 \mathrm{E}-8$ & $2.82 \pm 0.44 \mathrm{E}-7$ & $2.38 \pm 0.30 \mathrm{E}-7$ \\
\hline $\operatorname{Ar}^{+3}(4740)$ & $1.37 \pm 0.15 \mathrm{E}-7$ & - & $7.80 \pm 1.00 \mathrm{E}-8$ & - & & $3.75 \pm 0.80 \mathrm{E}-7$ & $2.08 \pm 0.50 \mathrm{E}-7$ \\
\hline \multirow[t]{2}{*}{$\mathrm{Ar}^{+4}(7006)$} & $3.21 \pm 0.40 \mathrm{E}-8$ & - & - & - & - & - & $1.34 \pm 0.20 \mathrm{E}-7$ \\
\hline & PN 16 & PN 18 & PN 19 & PN21 & H II 15 & Н II 9 & 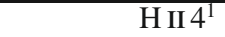 \\
\hline $\mathrm{T}(\mathrm{O} \mathrm{I}$ & $14390 \pm 720$ & $18000 \pm 1000$ & $20000 \pm 2000$ & $13668 \pm 1500$ & $11700 \pm 300$ & 12000 (adopt) & 12000 (adopt) \\
\hline $\mathrm{T}(\mathrm{N} \mathrm{I}$ & & & & & $12400 \pm 1500$ & & \\
\hline $\mathrm{N}(\mathrm{S} \mathrm{III})\left(\mathrm{cm}^{-3}\right)$ & $1140 \pm 200$ & $1420 \pm 400$ & $<100$ & $184 \pm 100$ & $187 \pm 100$ & $<100$ & \\
\hline N(Ar I & $5100 \pm 2000$ & & & & & & \\
\hline $\mathrm{He}^{+}$ & $23 \pm 0.10 \mathrm{E}-1$ & $.00 \mathrm{E}-2$ & $1.30 \pm 0.50 \mathrm{E}-1$ & $1.00 \pm 0.10 \mathrm{E}-1$ & $23 \pm 0.05 \mathrm{E}-2$ & $4.5 \pm 1.4 \mathrm{E}-2$ & $1.50 \pm 0.50 \mathrm{E}-2$ \\
\hline $\mathrm{He}^{++}(\mathrm{r}$ & $2.50 \pm 0.25 \mathrm{E}-2$ & $6.5 \pm 1.6 \mathrm{E}-2$ & $1.50 \pm 0.30 \mathrm{E}-2$ & $0.00 \mathrm{E}+00$ & $0.00 \mathrm{E}+00$ & $0.00 \mathrm{E}+00$ & $0.00 \mathrm{E}+00$ \\
\hline $\mathrm{O}^{+}(3727)$ & $1.73 \pm 0.50 \mathrm{E}-5^{3}$ & - & $1.64 \pm 0.40 \mathrm{E}-5^{3}$ & $5.5 \pm 1.5 \mathrm{E}-6$ & $4.31 \pm 0.30 \mathrm{E}-5$ & - & - \\
\hline $\mathrm{O}^{++}(5$ & $39 \pm 0.20 \mathrm{E}-4$ & $1.88 \pm 0.30 \mathrm{E}-5$ & $1.82 \pm 0.40 \mathrm{E}-5$ & $8.3 \pm 2.0 \mathrm{E}-5$ & $8.36 \pm 0.40 \mathrm{E}-5$ & - & - \\
\hline $\mathrm{N}^{+}(6$ & $0 \pm 0.20 \mathrm{E}-6$ & $2.13 \pm 0.40$ E-6 & $1.17 \pm 0.50 \mathrm{E}-6$ & $1.07 \pm 0.30$ E-6 & $1.23 \pm 0.20$ E- 6 & - & - \\
\hline $\mathrm{Ne}^{++}(3869)$ & $6 \pm 0.30 \mathrm{E}-5$ & $4.01 \pm 0.70$ E-6 & - & $1.51 \pm 0.40 \mathrm{E}-5$ & $1.54 \pm 0.20 \mathrm{E}-5$ & - & - \\
\hline$S$ & $12 \pm 0.60 \mathrm{E}-8$ & $1.58 \pm 0.15 \mathrm{E}-7$ & $6.7 \pm 1.1 \mathrm{E}-7$ & $1.44 \pm 0.40 \mathrm{E}-7$ & $2.72 \pm 0.30 \mathrm{E}-7$ & - & - \\
\hline $\mathrm{S}^{++}(6$ & $26 \pm 1.00 \mathrm{E}-7$ & - & $8.8 \pm 1.2 \mathrm{E}-7$ & $<3.00$ E-6 & $2.47 \pm 0.30 \mathrm{E}-6$ & - & - \\
\hline $\mathrm{Ar}^{++}(7$ & $2.57 \pm 0.30 \mathrm{E}-7$ & - & $2.26 \pm 0.60 \mathrm{E}-7$ & $3.37 \pm 0.50 \mathrm{E}-7$ & $6.23 \pm 0.15 \mathrm{E}-7$ & - & - \\
\hline $\mathrm{Ar}^{+3}(4740)$ & $3.99 \pm 0.40 \mathrm{E}-7$ & - & - & - & - & - & - \\
\hline
\end{tabular}

${ }^{1}$ Observed with Gemini; ${ }^{2}$ T(N II) for ionic abundances; ${ }^{3}$ [O II $] 7320$ for $\mathrm{O}^{+}$abundance; ${ }^{4}$ line intensity from Richer \& McCall (2007).

\subsection{Abundances in $\mathrm{HII}$ regions: Is the ISM in NGC 6822 chemically homogeneous?}

No conclusive evidence for chemical inhomogeneities in the present ISM of NGC 6822 has been found (Lee et al. 2006). To further analyze this, we compare our results for $\mathrm{H}$ II 15 with the other two best determined results for $\mathrm{HII}$ regions from the literature which are $\mathrm{HV}$, and $\mathrm{HX}$. Their abundances were taken from collisionally excited lines determinations by Peimbert et al. (2005). The three regions show, on average $\langle 12+\log (\mathrm{O} / \mathrm{H})\rangle=$ $8.06 \pm 0.04$. If we include the values reported by Lee et al. (2006) for their objects with [O III] $\lambda 4363$ measured (presented in Table 4), we still found a very chemically homogeneous galaxy, with an average abundance of $\langle 12+\log (\mathrm{O} / \mathrm{H})=8.08$ $\pm 0.06\rangle$. All these HII regions belong to the optical zone of NGC 6822, with H II 15 very near (de-projected distance 62 pc) to the galactic center proposed by Brandenburg \& Skillman (1998) while H V and HX are much to the North, at de-projected distances of about 1.01 and $1.08 \mathrm{kpc}$ from the center (half-way to the border of the $\mathrm{HI}$ disk), $\mathrm{H} 1$ is $1.78 \mathrm{kpc}$ to the North-West and $\mathrm{K} \alpha$ is at $1.30 \mathrm{kpc}$ to the West. Thus, our conclusion is that the present ISM is chemically homogeneous, at least in the $2 \mathrm{kpc}$ around the center. Other heavy elements like N, Ne, S and Ar are also very homogeneous for the three best observed $\mathrm{H}$ II regions, showing average values of $\langle\mathrm{N} / \mathrm{O}\rangle=0.05 \pm 0.02,\langle\mathrm{Ne} / \mathrm{O}\rangle=0.184$ $\pm 0.005,\langle\mathrm{~S} / \mathrm{O}\rangle=0.029 \pm 0.005$ and $\langle\mathrm{Ar} / \mathrm{O}\rangle=0.007 \pm 0.002$. The present ISM chemical abundances in NGC 6822 (based on the three best observed H II regions) are closer to those in the SMC than to those in the LMC (see Table 4), except for N/H which lies in the middle. The N/O abundance ratio of 0.05 is higher than the 0.03 value of both Clouds. Thus, NGC 6822 could be a more evolved galaxy than the Magellanic Clouds (similar results were obtained by Peimbert et al. 2005 from their C/O ratio).

\subsection{PN abundances}

For our PN sample, the abundances present a different situation than $\mathrm{H}$ II regions. In Fig. 2 we present the behavior of $12+\log (\mathrm{Ne} / \mathrm{H})$ vs. $12+\log (\mathrm{O} / \mathrm{H})$, and $12+\log (\mathrm{Ar} / \mathrm{H})$ vs. $12+\log (\mathrm{O} / \mathrm{H})$ abundances for all our PNe. The average values for $\mathrm{H}$ II regions are marked.

In the upper panel of this Figure, it is observed that the usual strong correlation between $\mathrm{Ne}$ and $\mathrm{O}$ abundances is very well defined for PNe and H II regions in NGC 6822. The slope of our linear fit is very near 1 and it is equal to the slopes reported for other galaxies; e.g., in the Milky Way a slope near 1 was reported by Henry (1989); Stasińska et al. (1998) report a very tight $\mathrm{Ne}-\mathrm{O}$ correlation (with a slope of about 1) for a PNe sample of several galaxies; the PNe in the LMC and SMC show slopes of 1.13 and 1.08 respectively (Leisy \& Dennefeld 2006), while in M 33 Magrini et al. (2009) report a slope of 0.90 for their PN sample. The average value $\langle\mathrm{Ne} / \mathrm{O}\rangle$, however, seems to vary with metallicity, being larger in richer galaxies (Wang \& Liu 2008).

Figure 2 shows that $\mathrm{O} / \mathrm{H}$ values in $\mathrm{PNe}$ are dispersed in a large range, from low-metallicity objects with $\mathrm{O} / \mathrm{H}$ abundances 0.5 dex poorer than the average of $\mathrm{HII}$ regions, up to 0.2 dex richer than this average. $\mathrm{PNe}$ are faint objects so their 
Table 4. Total abundances for PNe and H II regions in NGC 6822 and other galaxies ${ }^{1}$.

\begin{tabular}{|c|c|c|c|c|c|c|c|}
\hline Object & $\mathrm{He} / \mathrm{H}$ & $\mathrm{O} / \mathrm{H}$ & $\mathrm{N} / \mathrm{H}$ & $\mathrm{Ne} / \mathrm{H}$ & $\mathrm{S} / \mathrm{H}$ & $\mathrm{Ar} / \mathrm{H}$ & Other IDs ${ }^{2}$, comments $^{3}$ \\
\hline PN 12 & $0.113 \pm 0.009$ & $8.26 \pm 0.10$ & $7.57 \pm 0.13$ & $7.44 \pm 0.10$ & $6.62 \pm 0.15$ & $5.83 \pm 0.10$ & PN14-S14, young \\
\hline PN 16 & $0.148 \pm 0.010$ & $8.25 \pm 0.08$ & $7.16 \pm 0.20$ & $7.51 \pm 0.10$ & $6.02 \pm 0.20$ & $5.82 \pm 0.12$ & PN13-S16, young \\
\hline PN 7 & $0.107 \pm 0.015$ & $8.20 \pm 0.15$ & $6.92 \pm 0.17$ & - & $5.98 \pm 0.20$ & $5.96 \pm 0.13$ & PN2, young \\
\hline PN 6 & $0.096 \pm 0.005$ & $8.15 \pm 0.07$ & $7.36 \pm 0.15$ & $7.38 \pm 0.12$ & $6.20 \pm 0.29$ & $5.63 \pm 0.10$ & PN1, old \\
\hline PN 14 & $0.109 \pm 0.006$ & $8.12 \pm 0.08$ & $8.44 \pm 0.14$ & - & $5.89 \pm 0.30$ & $5.81 \pm 0.12$ & PN7-S33, Type I, young \\
\hline PN 21 & $0.106 \pm 0.012$ & $7.95 \pm 0.10$ & $7.24 \pm 0.15$ & $7.21 \pm 0.10$ & $<6.75$ & $5.80 \pm 0.12$ & PN12, young \\
\hline PN 5 & $0.094 \pm 0.015$ & $7.90 \pm 0.10$ & $8.36 \pm 0.20$ & - & $6.64 \pm 0.29$ & $5.88 \pm 0.15$ & PN3, Type I, young \\
\hline PN 4 & $0.090 \pm 0.005$ & $7.78 \pm 0.07$ & $7.48 \pm 0.12$ & $7.05 \pm 0.10$ & $6.22 \pm 0.15$ & $5.59 \pm 0.10$ & PN4, Type I?, old \\
\hline PN 10 & $0.111 \pm 0.010$ & $7.60 \pm 0.08$ & $6.30 \pm 0.13$ & $6.80 \pm 0.10$ & - & $5.20 \pm 0.15$ & PN19, old \\
\hline PN 19 & $0.145 \pm 0.040$ & $7.57 \pm 0.12$ & $6.42 \pm 0.15$ & - & $6.22 \pm 0.20$ & $5.63 \pm 0.15$ & PN9, old \\
\hline PN 18 & $<0.124$ & $7.49 \pm 0.15$ & - & $6.82 \pm 0.15$ & - & - & PN11, old \\
\hline$\langle$ non-type I $\rangle$ & $0.114 \pm 0.014$ & $7.92 \pm 0.18$ & $7.06 \pm 0.31$ & $7.17 \pm 0.20$ & $6.21 \pm 0.17$ & $5.68 \pm 0.15$ & Type I PNe excluded, $2 \sigma$ err \\
\hline Н II 15 & $0.090 \pm 0.005$ & $8.10 \pm 0.05$ & $6.56 \pm 0.08$ & $7.36 \pm 0.07$ & $6.49 \pm 0.10$ & $5.97 \pm 0.08$ & H II 8-S28 \\
\hline H V & $0.084 \pm 0.002$ & $8.08 \pm 0.03$ & $6.85 \pm 0.15$ & $7.35 \pm 0.03$ & $6.61 \pm 0.05$ & $5.84 \pm 0.03$ & PPR \\
\hline $\mathrm{HX}$ & $0.084 \pm 0.002$ & $8.01 \pm 0.05$ & $6.76 \pm 0.16$ & $7.27 \pm 0.05$ & $6.51 \pm 0.06$ & $5.84 \pm 0.05$ & PPR \\
\hline HI & - & $7.98 \pm 0.09$ & - & $7.34 \pm 0.19$ & - & - & LSV \\
\hline $\mathrm{K} \alpha$ & - & $8.00 \pm 0.04$ & $6.16 \pm 0.10$ & $7.51 \pm 0.10$ & - & $6.04 \pm 0.07$ & LSV \\
\hline $\mathrm{K} \beta$ & - & $8.18 \pm 0.07$ & - & $7.60 \pm 0.13$ & - & - & LSV \\
\hline KD28e & - & $8.19 \pm 0.05$ & $6.38 \pm 0.06$ & $7.64 \pm 0.09$ & - & $6.10 \pm 0.05$ & LSV \\
\hline$\langle$ H II regions $\rangle$ & $0.086 \pm 0.004$ & $8.06 \pm 0.04$ & $6.72 \pm 0.14$ & $7.33 \pm 0.05$ & $6.54 \pm 0.06$ & $5.88 \pm 0.07$ & 3 best observed obj., $2 \sigma$ err \\
\hline LMC-H II & 0.085 & 8.40 & 6.90 & 7.60 & 6.70 & 6.20 & D89, G99 \\
\hline LMC-PNe & 0.091 & 8.33 & 7.45 & 7.54 & 6.93 & 5.93 & non-type I, LD06 \\
\hline SMC-H II & 0.079 & 8.00 & 6.50 & 7.20 & 6.30 & 5.90 & D89, G99 \\
\hline SMC-PNe & 0.079 & 8.09 & 7.11 & 7.16 & 6.62 & 5.51 & non-type I, LD06 \\
\hline NGC3109-H II & 0.085 & 7.77 & 6.44 & 6.87 & 6.39 & 5.83 & PSR \\
\hline NGC3109-PNe & 0.087 & 8.16 & 7.49 & 7.18 & 6.26 & 5.66 & PSR \\
\hline Sun & 0.085 & 8.66 & 7.78 & 7.84 & 7.14 & 6.18 & Grevese et al. (2007) \\
\hline
\end{tabular}

${ }^{1}$ Heavy elements in $12+\log (\mathrm{X} / \mathrm{H})$, objects ordered by $12+\log (\mathrm{O} / \mathrm{H})$ values; ${ }^{2}$ IDs: PN\# from Leisy et al. (2005) and S\# from Killen \& Dufour (1982); ${ }^{3}$ Ref.: PPR: Peimbert et al. (2005); LSV: Lee et al. (2006), D89: Dennefeld (1989); G99: Garnett (1999); LD06: Leisy \& Dennefeld (2006); PSR: Peña et al. (2007).

uncertainties in abundances are larger than in the $\mathrm{H}$ II regions analyzed, however the dispersion is real, considering the uncertainties in abundance determinations. The richer objects, with $12+\log (\mathrm{O} / \mathrm{H})$ in the range 7.9-8.2, could be considered as having abundances similar to $\mathrm{H}$ II region abundances, but the objects at the low metallicity $\operatorname{limit}(12+\log (\mathrm{O} / \mathrm{H})<7.8)$ belong to an older less-enriched population.

The original $\mathrm{O}$ and $\mathrm{Ne}$ abundances in $\mathrm{PNe}$ can be perturbed, either by $\mathrm{O}$ destruction through the $\mathrm{ON}$-cycle in more massive progenitors, or when freshly synthesized $\mathrm{O}$ (and $\mathrm{Ne}$ ) is dredged up to the atmosphere of low metallicity PN progenitors (third dredge-up). The limit in metallicity for such an enrichment is still debated; thus, so far for NGC 6822, neither of these elements can be considered safe for testing the original chemical composition of PN progenitor stars and the use of other elements, such as $\mathrm{S}$ and Ar, is necessary.

For most of our objects, $\mathrm{S}$ and Ar were determined, although these elements have large uncertainties. In particular, the $S$ abundance has large uncertainties due to only $\mathrm{S}^{+}$and $\mathrm{S}^{++}$are observed, these ions being under-abundant in high excitation PNe. Besides, the icfs proposed by Kingsburgh \& Barlow (1994) depend on $\mathrm{O}^{+}$, also an under-abundant ion. Therefore, $\mathrm{S}$ abundances are not very reliable. On the other hand, Ar is better determined as the observed ions $\left(\mathrm{Ar}^{++}\right.$and $\left.\mathrm{Ar}^{+3}\right)$ are the most abundant species in high excitation $\mathrm{PNe}$ and the ifcs are small. In Fig. 2 (lower panel), Ar vs. O abundances are presented. Contrary to what happens with $\mathrm{Ne}$, there is no obvious Ar to $\mathrm{O}$ relation, except for an increasing trend which is expected and that also appears in the PN samples of the other galaxies mentioned. The important fact here is that no PN presents an $\mathrm{Ar} / \mathrm{H}$ abundance ratio larger than the average value of $\mathrm{H}$ II regions.
Besides, PNe can be divided roughly in two groups: one with an $\mathrm{Ar} / \mathrm{H}$ ratio similar to $\mathrm{H}$ II regions (all these objects also present large $\mathrm{O} / \mathrm{H}$ ratios) and another with low $\mathrm{Ar} / \mathrm{H}$ values. In the following, we will discuss PN abundances in terms of these two groups.

\subsection{The $N$ abundances and Type I PNe}

In Table 4 we find that all $\mathrm{PNe}$, but two, show $\mathrm{N}$-enrichment relative to the average value for H II regions. Similar results are found for all galaxies and this is usually interpreted as a result of the different dredge-up episodes in the PN progenitor star, where some products from nucleosynthesis (mainly $\mathrm{He}, \mathrm{C}$ and $\mathrm{N}$ ) are brought to the surface. The $\mathrm{N}$-enrichment in PNe indicates that these objects are a major source of $\mathrm{N}$ production in a galaxy, but for NGC 6822, such nitrogen has not enriched the ISM yet.

$\mathrm{N}$-enrichment occurs at the stellar surface during the AGB dredge-up events and it has been attributed to the $\mathrm{CN}$ cycle where original $\mathrm{C}$ is transformed into $\mathrm{N}$ (for the more massive progenitors, $\mathrm{O}$ also can be transformed to $\mathrm{N}$ through the ON cycle, e.g. Henry 1990, and nebulae show depleted O). In addition, very $\mathrm{N}$-rich nebulae (usually named Type I) can be produced by the more massive progenitors that can experience envelope-burning conversion to nitrogen of dreged-up primary carbon. Different criteria have been proposed to define these particularly N-enriched PNe. Peimbert (1978) classified as Type I PNe those galactic nebulae with $\mathrm{He} / \mathrm{H}>0.125$ and N/O $>0.5$, while Kingsburgh \& Barlow (1994) defined Type I PNe as those nebulae having $\mathrm{N}$ abundances that exceed the total $\mathrm{C}+\mathrm{N}$ abundance of $\mathrm{H}$ II regions in the same galaxy and 


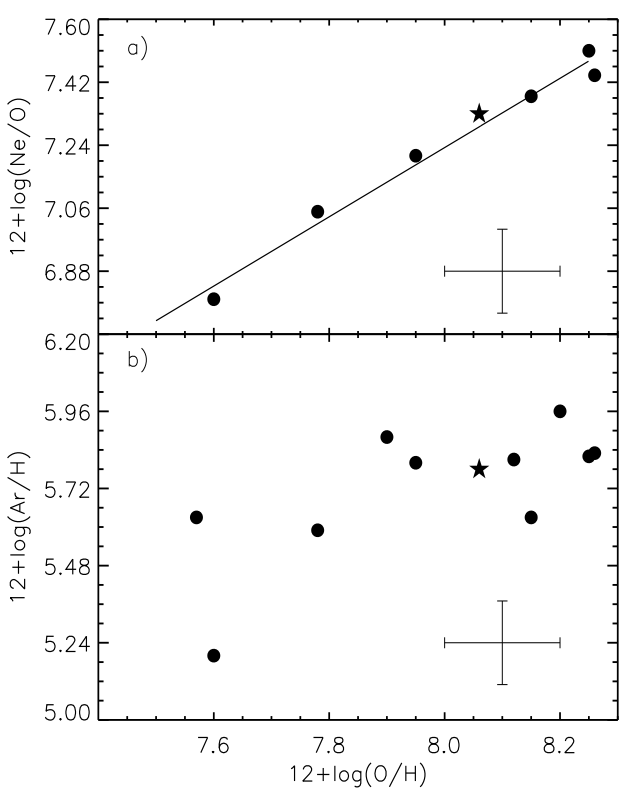

Fig. 2. The behavior of $12+\log (\mathrm{Ne} / \mathrm{H})$ and $12+\log (\mathrm{Ar} / \mathrm{H})$ vs. $12+\log (\mathrm{O} / \mathrm{H})$. Filled dots are $\mathrm{PNe}$ and the star is the average value for $\mathrm{H}$ II regions. The tight linear correlation between $\mathrm{Ne}$ and $\mathrm{O}$ corresponds to $12+\log (\mathrm{Ne} / \mathrm{H})=0.99(12+\log (\mathrm{O} / \mathrm{H}))-0.72$, with a correlation coefficient $R^{2}=0.98$ (after removing the more deviant point $\mathrm{PN} 18$ ). $\mathrm{Ar} / \mathrm{H}$ determination is much more uncertain and the correlation is not as clear. But it is found that no $\mathrm{PNe}$ has $\mathrm{Ar} / \mathrm{H}$ larger than the $\mathrm{H}$ II region value, which is not the case for $\mathrm{Ne} / \mathrm{H}$ and $\mathrm{O} / \mathrm{H}$.

suggested a limit of N/O $>0.8$ for our galaxy, independently of the He abundance. These criteria require some corrections for PNe in low-metallicity galaxies, e.g., Leisy \& Dennefeld (1996) suggest a limit $\mathrm{N} / \mathrm{O}>0.25$ for Type I PNe in the Magellanic Clouds. In our case we will employ the Kingsburgh \& Barlow criteria, by considering that Peimbert et al. (2005) found $\log$ $\mathrm{C} / \mathrm{O}=-0.31 \pm 0.13$ for $\mathrm{H} \mathrm{V}$ in NGC 6822 and that $\mathrm{N} / \mathrm{O}=0.05$ in $\mathrm{H}$ II regions. This would imply that a Type I PN in this galaxy should have $\mathrm{N} / \mathrm{O} \geq 0.49$ (there is a large margin of uncertainty in this value as, considering Peimbert et al. 2005 uncertainties for $\mathrm{C} / \mathrm{O}$, the limit of N/O for a Type I PN is in the range from 0.36 to 0.66 ). Thus, in our sample we clearly find two extreme Type I PNe (PN 5 and PN 14) with N/O $>2$, and a possible third Type I PN, PN 4, with N/O $\sim 0.50$.

The $\mathrm{O}$ and $\mathrm{Ar}$ abundances in the extreme Type I PNe, PN 14 and PN 5, are equal, within uncertainties, to the average values of $\mathrm{H}$ II regions, thus confirming that they are young objects. The third Type I candidate, PN 4, which is the brightest PN observed in this galaxy, requires a closer look. All its elemental abundances, but $\mathrm{N}$, are about a factor of 2 lower than the averages in $\mathrm{HII}$ regions, therefore this object should have emerged in a less enriched medium, a few Gyrs ago, and probably its progenitor is not very massive. Also, it should be taken into account that the $\mathrm{N} / \mathrm{O}$ abundance ratio is determined via the $\mathrm{N}^{+} / \mathrm{O}^{+}$ionic abundance ratio which is very temperature dependent. For PN 4 we have determined both $\mathrm{T}(\mathrm{O}$ III) and $\mathrm{T}(\mathrm{N}$ II) electron temperatures (although T(N II) has a large uncertainty) then, to test our abundance ratio, we calculated $\mathrm{N}^{+}$and $\mathrm{O}^{+}$ionic abundances using $\mathrm{T}\left(\mathrm{N}\right.$ II). We found a $\mathrm{N}^{+} / \mathrm{O}^{+}$abundance ratio of 0.45 instead of 0.50 . Then, according to our criteria, PN 4 is at the limit and can be considered only marginally as a Type I PN.

\subsection{Non Type I PNe}

Among the non Type I nebulae (those that are not N-rich), there are a few (PN 7, PN 12, and PN 16) showing O/H abundance ratios about $0.15-0.20$ dex higher than $\mathrm{H}$ II regions. Also, the $\mathrm{Ne} / \mathrm{H}$ values in PN 12 and PN 16 seem to be slightly enhanced, relative to $\mathrm{H}$ II regions. On the other hand, the three nebulae present Ar abundances equal, within uncertainties, to $\mathrm{H}$ II regions (see Table 4 and Fig. 2). These PNe then could be $\mathrm{O}$ and $\mathrm{Ne}$-enriched nebulae, via the third dredge-up. However, the effect is small and other explanations are possible, for example, $\mathrm{O}$ locked in dust grains in $\mathrm{H}$ II regions. Based on their Ar abundance, we will consider these nebulae as belonging to the young PNe.

Three non-type I objects, PN 10, PN 18 and PN 19, are O, $\mathrm{Ne}$ and Ar poorer than HII regions by factors larger than 2 . Together with the Type I candidate, PN 4, these objects correspond to bona-fide low-metallicity PN progenitors and they belong to an older population formed in a less enriched medium. In this group we will also include PN 6, which has an $\mathrm{O} / \mathrm{H}$ similar to $\mathrm{H}$ II regions but a much lower Ar abundance.

\subsection{Comparison with samples in other galaxies}

In Table 4 we present the average abundances values for the three best determined $\mathrm{H}$ II regions and the whole sample of non-type I PNe. For comparison, we have included the respective values for the the Magellanic Clouds as derived by Leisy \& Dennefeld (1996, 2006) and also the abundances derived for the low metallicity irregular galaxy NGC 3109 and the solar values. In general, the chemical abundance pattern of PNe in NGC 6822 follows closely the behavior of PNe in the Magellanic Clouds. The average abundance values of heavy elements in non-type I PNe in NGC 6822 are very similar to the ones in the SMC. In this case (and contrary to $\mathrm{H}$ II regions) the $\mathrm{N} / \mathrm{H}$ ratio also shows similarity with the SMC, indicating similar nebular enrichment due to stellar nucleosynthesis. Differently to PNe in NGC 3109, the PN sample in NGC 6822 does not show O-enrichment relative to $\mathrm{H}$ II regions. For NGC 3109, this O-enrichment could be attributed to newly synthesized $\mathrm{O}$ dredged-up by convection in the progenitor envelopes. As already said, such an enrichment happens mainly in low metallicity environments $(12+\log (\mathrm{O} / \mathrm{H})=7.77$ in $\mathrm{H}$ II regions of NGC 3109). Apparently the metallicity of NGC 6822 is not low enough to detect such an event.

Non Type I PNe in NGC 6822 appear richer in He than in the other galaxies but this could be an artifact due to a pair of apparently very He-rich nebulae: PN 16 and PN 19. The latter one has a large uncertainty in the $\mathrm{He} / \mathrm{H}$ determination and if it is excluded from the average, we obtain an $\mathrm{He} / \mathrm{H}$ average value of $0.096 \pm 0.006$, still large but, within uncertainties, it is similar to the value in the LMC. Interestingly, contrary to expectations, the two extreme Type I PN are not particularly He-rich.

\section{Chemical enrichment in NGC 6822. A preliminary evolution model}

\subsection{Observational constraints}

In Sect. 4 we present the chemical abundances of several H II regions taken from diverse authors, including our H II 15 determinations, as derived from collisionally excited lines. Those $\mathrm{H}$ II regions are located in different parts of the optical area and we have found no evidence of chemical inhomogeneities in this galaxy. For that reason we choose the average $\langle 12+\log (\mathrm{O} / \mathrm{H})\rangle$ $=8.06 \pm 0.04$ as representative of the current $\mathrm{O} / \mathrm{H}$ abundance 
ratio of the whole galaxy and we have computed several chemical evolution models of NGC 6822 to reproduce this $\mathrm{O} / \mathrm{H}$ value. Notice that no $\mathrm{O}$ locked in dust grains has been considered.

Abundances of PNe in our sample will be used as additional constraints. As discussed in Sect. 4, several PNe in our sample belong to the young population in this galaxy and their average $\mathrm{O} / \mathrm{H}$ ratio is similar to the one of $\mathrm{H}$ II regions. Their other elements are also similar to $\mathrm{H}$ II regions, therefore this PN sample does not provide further constraints, but there is a sample of older PNe, with much lower $\mathrm{O}, \mathrm{Ne}$ and Ar that will help to test the model predictions at the time of formation of their progenitors. The elements used to constrain the model should be those that clearly represent the initial abundances in the progenitors. It is known that nucleosynthesis and convective processes in LIMS perturbed mainly $\mathrm{He}, \mathrm{C}$, and $\mathrm{N}$ abundances in PNe. We have not determined $\mathrm{C}$ abundances for our $\mathrm{PNe}$, but we do derive $\mathrm{He}$ and $\mathrm{N}$ abundances, which will not be used as observational constraints in our model. In addition, as explained in the introduction, the $\mathrm{O} / \mathrm{H}$ ratio could have been affected by processes such as ON cycle or third dredge-up, therefore it should be cautiously considered as a confident tracer of the original $\mathrm{O}$ abundance. On the other hand, the production of $\mathrm{S}, \mathrm{Cl}$, and $\mathrm{Ar}$ by LIMS is negligible, so these elements can be considered as important observational constraints of the ISM in the past. For our PN sample we only have $\mathrm{S}$ and Ar abundances, but only Ar determinations are reliable. Thus, for our chemical evolution modelling we will rest mainly on Ar abundances to support our conclusions

Thus, based mainly on their Ar abundances, we have divided the PNe of Table 4 in two groups: "young" and "old". PN 5, PN 7, PN 12, PN 14, PN 16 and PN 21, which show $\langle 12+\log (\mathrm{O} / \mathrm{H})\rangle=8.10 \pm 0.10$ and $\langle 12+\log (\mathrm{Ar} / \mathrm{H})\rangle=5.85 \pm$ 0.06 , are young objects. PN 4, PN 6, PN 10, PN 18, and PN 19, with $\langle 12+\log (\mathrm{O} / \mathrm{H})\rangle=7.71 \pm 0.20$ and $\langle 12+\log (\mathrm{Ar} / \mathrm{H})\rangle=$ $5.50 \pm 0.13$, are PNe with old progenitors. Following Allen et al. (1998), we will assume ages between 1 to 3 Gyr for the young PN population and between 3 and 9 Gyr for the old one. The present age of the galaxy is assumed to be $13.5 \mathrm{Gyr}$.

In the next section, we explain briefly the code we have developed to compute chemical evolution models. A preliminary model has been computed for NGC 6822, which fits quite well some of the constraints mentioned above. In a following paper (Hernández-Martínez et al., in preparation) we will discussed extensively other more detailed models, with their assumptions and results.

\subsection{Chemical evolution models}

\subsubsection{Antecedents}

Carigi et al. (2006, hereafter CCP) computed chemical evolution models for NGC 6822 using the CHEVO code, which follows the lifetime of each star formed. These models were built to reproduce the gaseous mass of the galaxy and the abundance value $12+\log (\mathrm{O} / \mathrm{H})=8.42 \pm 0.06$, as determined by Peimbert et al. (2005) for Hubble $\mathrm{V}(\mathrm{HV})$, the brightest $\mathrm{H}$ II region of the galaxy. The Peimbert et al. $\mathrm{X}_{\mathrm{i}} / \mathrm{H}$ abundance ratios are higher than ours, because their determinations are from recombination lines or collisionally excited lines considering temperature fluctuations, $t^{2}>0$ and dust correction. CCP also considered as constraints the $\mathrm{C} / \mathrm{O}, \mathrm{N} / \mathrm{O}$ and $\mathrm{Fe} / \mathrm{O}$ abundance ratio, also derived with $t^{2}>0$.

The code used by CCP allows the evolution of 6 elements only. Then they computed models for a few chemical elements.
Their model results could only be compared with observational data at the present time because that work was based on abundances exclusively from $\mathrm{H}$ II regions. In this work, we are able to explore a more precise model as we compute a model for all the observed chemical elements for which stellar yields are available, and we study the whole chemical evolution of NGC 6822 comparing our model results with current and past observational data from $\mathrm{H}$ II regions and $\mathrm{PNe}$.

\subsubsection{Our code}

The code developed by one of us (L.H.-M.), and applied to NGC 6822, takes into account the delayed contribution of all LIMS as represented by one "average" star that enriches the interstellar medium, while it is considered that all massive stars (MS) enrich the ISM instantaneously, in a similar way as presented by Franco \& Carigi (2008). The new code was developed considering a general star formation, initial mass function, infalls, outflows, and stellar yields for 27 elements. Moreover this code includes the progenitors of Type Ia supernovae (SNIa), the main producers of Fe, unlike models by Franco \& Carigi (2008).

Our model assumes:

i) the star formation history ( $\mathrm{SFH}$ ) obtained by $\mathrm{CCP}$ that reproduces the photometric properties of NGC 6822;

ii) the initial mass function by Kroupa et al. (1993, here after KTG IMF) between 0.1 and $40 M_{\odot}$, in order to reproduce the current $12+\log (\mathrm{O} / \mathrm{H})=8.06 \pm 0.04$;

iii) the infall computed by CCP predicted by a $\Lambda$ CDM cosmology;

iv) a well-mixed outflow during $5.3 \mathrm{Gyr}$, in order to reproduce the present-day gas mass observed in NGC 6822, (1.98 \pm $0.02) \times 10^{8} M_{\odot}($ see CCP) and; v) a set of metallicity $Z$ dependent yields for 27 elements formed by massive star yields, low and intermediate mass star yields, and Type Ia SN yields.

For massive stars $\left(M>11 M_{\odot}\right)$ we consider yields by pre-SN stage and SN stage. The pre-SN yields are taken from the Geneva group (Hirshi 2007; Meynet \& Maeder 2002; Hirshi et al. 2005). The SN yields are taken from Woosley \& Weaver (1995) adopting their B models, for 12 to $30 M_{\odot}$ and their $\mathrm{C}$ models, for 35 to $40 M_{\odot}$. We combine Geneva yields with Woosley \& Weaver yields using the prescription explained by Carigi \& Hernandez (2008). The SN explosions do not contribute to He, C, N, and $\mathrm{O}$ yields, but they do to heavier elements, like $\mathrm{Ne}, \mathrm{S}, \mathrm{Cl}$, Ar, and Fe.

For LIMS $\left(1<M<6 M_{\odot}\right.$ ) we adopted the new yields by Karakas \& Lattanzio (2007). Their models were calculated from the zero-age main sequence to near the end of the thermallypulsing asymptotic giant branch including the PN phase. Their code is quite complete and considers the core He-flash, the third dredge-up, and the hot bottom burning, during a total number of thermal pulses between approximately 20 and 100, that increases with the initial mass and decreases with the initial metallicity. They assume a complex CNO treatment and their models predict $\mathrm{O}$ production only for very metal-poor LIMS $(Z=$ $\left.1.0 \times 10^{-4}=0.005 Z_{\odot}\right)$. For the rest of initial stellar metallicities, their models predict $\mathrm{O}$ destruction, opposite to Marigo's model (Marigo 2001). They do not considered scaled-solar compositions and therefore their yields are more reliable to study objects with chemical abundances similar to the Magellanic Clouds, like NGC 6822. 

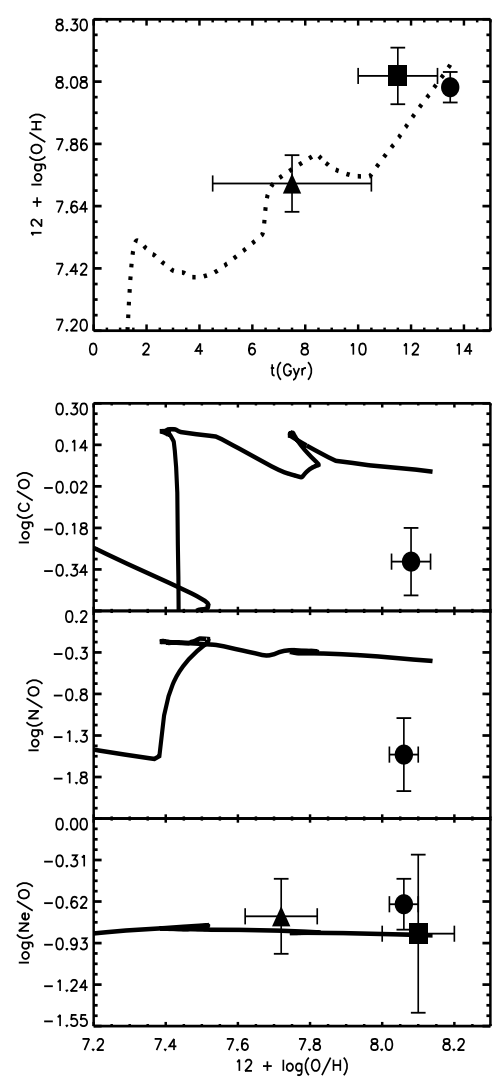
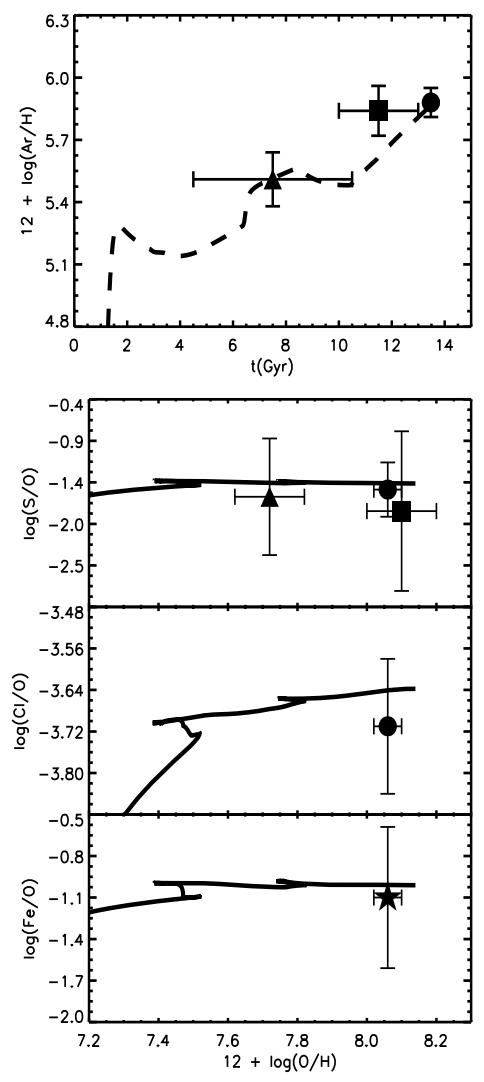

Fig. 3. Evolution of $12+\log (\mathrm{O} / \mathrm{H})$ and $12+\log (\mathrm{Ar} / \mathrm{H})$ vs. time and $\mathrm{C} / \mathrm{O}, \mathrm{N} / \mathrm{O}, \mathrm{Ne} / \mathrm{O}$, $\mathrm{S} / \mathrm{O}, \mathrm{Cl} / \mathrm{O}, \mathrm{Ar} / \mathrm{O}$ and $\mathrm{Fe} / \mathrm{O}$ vs. $12+\log (\mathrm{O} / \mathrm{H})$ as predicted by one of our best chemical evolution models for NGC 6822 (discussed in the text). The observational data show the average abundance values for $\mathrm{HII}$ regions (filled circles), young $\mathrm{PNe}$ (filled squares), old $\mathrm{PNe}$ (filled triangles). $\mathrm{O}, \mathrm{N}, \mathrm{Ne}, \mathrm{S}$ and $\mathrm{Ar}$ observational abundances of $\mathrm{HII}$ regions and $\mathrm{PNe}$ are from Table 4 (this work). $\mathrm{C} / \mathrm{O}$ and $\mathrm{Cl} / \mathrm{O}$ ratios were adopted from Peimbert et al. (2005). Data shown in the $\mathrm{Fe} / \mathrm{O}$ graph (lower right) correspond to the average $\mathrm{Fe} / \mathrm{H}$ ratio of two A-type supergiants reported by Venn et al. (2001) and the $\mathrm{O} / \mathrm{H}$ value of this work.
Since the mentioned yields for high and low mass stars do not cover the $6-11 M_{\odot}$ range, we extrapolate the MS and LIMS yields to $7.5 M_{\odot}$.

Iron abundance in a galaxy is mainly produced by Type Ia supenovae. A SNIa originates from a binary system of LIMS, after the most massive member becomes a C-O white dwarf and accretes material from its companion at any evolutionary stage (main sequence, red giant, or $\mathrm{C}-\mathrm{O}$ white dwarf). But the exact physical mechanisms are not clear and consequently the time delay of that type of $\mathrm{SN}$ are still unknown.

For SNIa, we assume that a fraction, $A_{\text {bin }}$, of the stars with masses between 3 and $15 M_{\odot}$ make up binary systems and all of those systems become SNIa. All Type Ia supernovae of each stellar generation enrich the interstellar medium 1 Gyr after formation of SNIa progenitors, according to Fig. 1 of Mannucci (2007). Each SNIa, independent of the initial metallicity, ejects heavy elements, mainly Fe, with an efficiency (yield) given by the model W7 from Nomoto et al. (1997).

The fraction $A_{\text {bin }}$ is a free parameter of the model and it is obtained in order to reproduce the average Fe observed in 2 stars of NGC $6822(\langle[\mathrm{Fe} / \mathrm{H}]\rangle=-0.49 \pm 0.22$ in solar units, Venn et al. 2001). Based on all previous assumptions, we get $A_{\text {bin }}=0.01$, that is, $1 \%$ of $3-15 M_{\odot}$ stars become SNIa.

\subsubsection{The results}

Our model, presented in Fig. 3, was tailored to reproduce the current $\mathrm{O} / \mathrm{H}$ value as given by $\mathrm{H}$ II regions (values from Table 4). It is equivalent to Model $7 \mathrm{~L}$ by $\mathrm{CCP}$, except that they assumed a KTG IMF with a mass upper-limit equal to $60 M_{\odot}$, while our model needs to reduced this limit to $40 M_{\odot}$, in order to match a lower current $\mathrm{O} / \mathrm{H}$.
In Fig. 3, the $12+\log (\mathrm{O} / \mathrm{H})$ and $12+\log (\mathrm{Ar} / \mathrm{H})$ evolution with time is shown; both elements behave very similarly with time. A fast enrichment is predicted during the first $2 \mathrm{Gyr}$, because $\mathrm{Ar}$ and $\mathrm{O}$ are produced mainly by MS. The enrichment is followed by a light fall (plateau) up to $6 \mathrm{Gyr}$, and then a second fast enrichment occurs, particularly accelerated in the last few Gyr, imitating the time dependence of SFH. The observational data for different objects (H II regions, young and old $\mathrm{PNe}$, and A-type stars) are also shown in Fig. 3.

We find that the predicted evolution of $\mathrm{O} / \mathrm{H}$ and $\mathrm{Ar} / \mathrm{H}$ for $t<7$ Gyr agrees with the average abundances of old PNe, considered to be $6 \pm 3$ Gyr old. This epoch coincides with the beginning of the second fast enrichment. Moreover, the recent evolution of $\mathrm{O} / \mathrm{H}$ and $\mathrm{Ar} / \mathrm{H}$ matches the average abundances of young PNe, assumed to be $2 \pm 1$ Gyr old (filled square in Fig. 3). The observational point (filled square) seems slightly deviated from the model line, but taking into account the large uncertainty in $\mathrm{PN}$ age, we consider that the model reproduces this point. Since $\mathrm{O} / \mathrm{H}$ and $\mathrm{Ar} / \mathrm{H}$ evolution depends mainly on star formation rate and the agreement with observations is good, we conclude the SFH inferred by photometric properties and assumed in this work truly represent the star formation history in NGC 6822.

Since our model assumes that most of the LIMS that formed during the evolution of the galaxy do not produce $\mathrm{O}$ and that the model reproduces well both $\mathrm{O}$ and $\mathrm{Ar}$ abundances in $\mathrm{PNe}$, it is apparent that $\mathrm{O}$ has not been perturbed in the PN progenitors of NGC 6822. Therefore, there is no evidence for significant third dredge-up affecting the $\mathrm{O}$ abundance at this metallicity.

In Fig. 3 we also present $\log \left(\mathrm{X}_{\mathrm{i}} / \mathrm{O}\right)$ vs. $12+\log (\mathrm{O} / \mathrm{H})$ behavior for different elements $\mathrm{X}_{\mathrm{i}}(\mathrm{C}, \mathrm{N}, \mathrm{Ne}, \mathrm{S}, \mathrm{Cl}$, and $\mathrm{Fe})$ obtained from our model. We have chosen to represent element abundance ratios as a function of $\mathrm{O}$ because this is a common way to display abundances in photoionized nebulae and in evolution modelling. 
For this figure, we have taken $\log (\mathrm{C} / \mathrm{O})=-0.31 \pm 0.13$ and $\log (\mathrm{Cl} / \mathrm{O})=-3.71 \pm 0.10$ from Peimbert et al. (2005), while $\log (\mathrm{Fe} / \mathrm{H})=-5.04 \pm 0.20$ is from Venn et al. (2001). This value, combined with our $\mathrm{O} / \mathrm{H}$ value from $\mathrm{H}$ II regions, gives a presentday abundance ratio of $\log (\mathrm{Fe} / \mathrm{O})=-1.1 \pm 0.5$. The figures show that for all the elements, but $\mathrm{N}$ and $\mathrm{C}$, the $\mathrm{X}_{\mathrm{i}} / \mathrm{O}$ vs. $\mathrm{O} / \mathrm{H}$ evolutions predicted by the model match very well with $\mathrm{H}$ II region and PN data within one $\sigma$ errors.

The predicted present-day $\mathrm{C} / \mathrm{O}$ and N/O ratios are $\sim 0.4$ and $1.0 \mathrm{dex}$, respectively, higher than the observed values. The $\mathrm{C} / \mathrm{O}$ discrepancy is complex because the value we are using was computed by Peimbert et al. (2005) from recombination lines (RL), while in this work we are using abundances from collisionally excited lines (CEL). However Peimbert et al. (2005) argue that the $\mathrm{C} / \mathrm{O}$ ratio does not depend on the temperature structure of the nebula, and thus $(\mathrm{C} / \mathrm{O})_{\mathrm{CEL}}=(\mathrm{C} / \mathrm{O})_{\mathrm{RL}}$, in which case the discrepancy should be due to other causes. Moreover, the dust correction considered by Peimbert et al. does not change their $\mathrm{C} / \mathrm{O}$ value, because that correction increases the $\mathrm{C} / \mathrm{H}$ and $\mathrm{O} / \mathrm{H}$ ratios by 0.10 and 0.08 dex, respectively.

Part of the problem with both elements is that we have reduced the mass-upper limit of KTG IMF to $40 M_{\odot}$, in order to match the low current $\mathrm{O} / \mathrm{H}$ obtained for $\mathrm{H}$ II regions. As $\mathrm{C}$ and $\mathrm{N}$ are mainly produced by LIMS, these elements are not reduced equivalently. In addition, at least for the case of $\mathrm{N}$, the yields by Karakas \& Lattanzio (2007) are higher than those provided by other authors, mainly for stars with $M>5 M_{\odot}$ and intermediate metallicity $(Z=0.008)$, thus providing a higher $\mathrm{N}$ abundance. This problem will be discussed in detail in Hernández-Martínez et al. (in preparation) where a set of models will be presented.

\section{Conclusions}

From MOS spectroscopy performed with 8-m class telescopes we derived chemical compositions for $11 \mathrm{PNe}$ and $1 \mathrm{H}$ II region in NGC 6822. From this, our results are the following:

a) Combining our HII region abundance with other well determined $\mathrm{H}$ II region values from the literature, we confirm that the present ISM is chemically homogeneous, at least in the central $2 \mathrm{kpc}$ of NGC 6822. The present average oxygen abundance obtained is $\langle 12+\log (\mathrm{O} / \mathrm{H})\rangle=8.06 \pm 0.04$.

b) PNe abundances fall in a larger range of abundances than $\mathrm{H}$ II regions. According to their $\mathrm{Ar} / \mathrm{H}$ abundances, we identify a young PN population with abundances similar to $\mathrm{H}$ II regions and an older population with abundances a factor of two lower.

c) All PNe present $\mathrm{N}$-enrichment relative to $\mathrm{H}$ II regions. Two of them are extremely N-rich (Type I PNe), and a third one, from the old population, also presents $\mathrm{N}$-enrichment compatible with a Type I PN.

d) $\mathrm{Ne}$ and $\mathrm{O}$ abundances in our objects, follow a very tight linear correlation with a slope near 1 . Some young non Type I PNe seem to have suffered slight $\mathrm{O}$ and $\mathrm{Ne}$ enrichment, previous to nebular ejection, showing abundance values slightly higher than $\mathrm{H}$ II regions. This effect however could be attributed to other causes, for example dust in $\mathrm{H}$ II regions. Interestingly, the old PN population, with $12+\log (\mathrm{O} / \mathrm{H}) \leq 7.8$, does not seem to be O-enriched compared with the predictions of our chemical evolution model. Thus, the third dredge-up of $\mathrm{O}$ did not occur in the $\mathrm{PNe}$ of NGC 6822. This would indicate that for a significant Oenrichement, a lower metallicity is necessary. e) The pattern of PNe chemical abundances in NGC 6822 follows closely that of the SMC, where a mixture of populations also is found.

For the first time, thanks to PN abundance determinations, a complete evolution model of NGC 6822 is obtained. The model, tailored to match the present $\mathrm{O} / \mathrm{H}$ abundance ratio determined in this work, matches the abundance ratios of heavy elements produced mainly by massive stars, and predicts well the behavior of most elements observed in the young and old PN populations. The model however needs some refinements in order to match $\mathrm{C}$ and $\mathrm{N}$ abundances, mainly produced by LIMS.

Based on galactic formation given by a $\Lambda \mathrm{CDM}$ cosmology and a star formation history increasing and bursting during the whole evolution of the galaxy, we infer the following characteristics for the chemical history of NGC 6822:

i) significant gas-mass loss occurred during the first $5.3 \mathrm{Gyr}$;

ii) no star more massive than $40 M_{\odot}$ was formed, and;

iii) one percent of all 3-15 $M_{\odot}$ stars became binary system progenitors to SNIa.

Acknowledgements. Invaluable comments and support by Manuel Peimbert and Michael Richer are deeply appreciated. M. Peña is grateful to DAS, Universidad de Chile, for hospitality during a sabbatical stay when part of this work was performed. L.H.-M. benefited from the hospitality of the Departamento de Astronomía, Universidad de Chile for this work. L.H.-M. received a scholarship from CONACYT-México and DGAPA-UNAM. J.G.-R. is supported by a postdoctoral grant from UNAM. M.P. gratefully acknowledges financial support from FONDAP-Chile and DGAPA-UNAM. This work received financial support from CONACYT-México (grants \#43121, \#46904 and \#60354) and DGAPAUNAM (grants IN-114805 and IN-112708).

\section{References}

Allen, C., Carigi, L., \& Peimbert, M. 1998, ApJ, 494, 247

Bauman, R. P., Porter, R. L., Ferland, G. J., et al. 2005, ApJ, 628, 541

Brandenburg, H. J., \& Skillman, E. D. 1998, BAAS, 30, 1354

Carigi, L., \& Hernandez, X. 2008, MNRAS, 390, 582

Carigi, L., Colin, P., \& Peimbert, M. 1999, ApJ, 514, 787

Carigi, L., Colin, P., \& Peimbert, M. 2006, ApJ, 644, 924 (CCP)

Costa, R. D. D., de Freitas Pacheco, J. A., \& Idiart, T. P. 2000, A\&AS, 145, 467

de Blok, W. G. J., \& Walter, F. 2000, ApJ, 537, 95

de Blok, W. G. J., \& Walter, F. 2006, AJ, 131, 343

Demers, S., Batinelli, P., \& Artigau, E. 2006, A\&A, 456, 905

Dennefeld, M. 1989, Recent development of Magellanic Clouds research, ed. K.

S. de Boer, F. Spite, \& G. Stasinska, 107

Dufour, R. J., \& Talent, D. L. 1980, ApJ, 235, 22

Fisher, J. R., \& Tully, R. B. 1979, AJ, 84, 62

Franco, I., \& Carigi, L. 2008, RMAA, 44, 311

Garnett, D. 1999, New Views of the Magellanic Clouds, ed. Y.-H. Chu, N. B. Suntzeff, J. E. Hesser, \& D. A. Bohlender, IAU Symp. 190, 266 Gieren, W., Pietrzyński, G., Nalewajko, K., et al. 2006, ApJ, 647, 1056 Grevesse, N., Asplund, M., \& Sauval, A. J. 2007, SSRv, 130, 105 Henry, R. B. C. 1989, MNRAS, 241, 453

Henry, R. B. C. 1990, ApJ, 356, 229

Hernández-Martínez, L., \& Peña, M. 2009, A\&A, 495, 447 (Paper I)

Herwig, F. 2004, ApJS, 155,651

Hidalgo-Gámez, A. M., Masegosa, J., \& Olofsson, K. 2001, A\&A, 369, 797

Hirschi, R. 2007, A\&A, 461, 571

Hirschi, R., Meynet, G., \& Maeder, A. 2005, A\&A, 433, 1013

Hodge, P., Smith, T., Eskridge, P., MacGilivray, H., \& Beard, S. 1991, ApJ, 379, 621

Karakas, A., \& Lattanzio, J. C. 2007, PASA 24, 103

Killen, R. M., \& Dufour, R. J. 1982, PASP, 94, 444

Kingdon, J., \& Ferland, G. J. 1995, ApJ, 442, 714

Kingsburgh, R., \& Barlow, M. 1994, MNRAS, 271, 257

Kniazev, A. Y., Grebel, E. K., Pustilnik, S. A., Pramskij, A. G., \& Zucker, D. B. 2005, AJ, 130, 1558

Kroupa, P., Tout, C. A., \& Gilmore, G. 1993, MNRAS, 262, 545 (KTG)

Lee, H., Skillman, E. D., \& Venn, K. A. 2006, ApJ, 642, 813

Leisy, P., \& Dennefeld, M. 1996, A\&ASS, 116, 95

Leisy, P., \& Dennefeld, M. 2006, A\&A, 456, 451

Leisy, P., Corradi, R. L. M., Magrini, L., et al. 2005, A\&A, 436, 437 
Magrini, L., Leisy, P., Corradi, R. L. M., et al. 2005, A\&A, 443, 115 Magrini, L., Stanghellini, L., \& Villaver, E. 2009, ApJ, 696, 729

Mannucci 2007, Multifrequency behaviour of high energy cosmic sources, ed. F. Giovannelli, \& L. Sabau-Graziati, ChJAA [arXiv: 0708 . 0472]

Marigo, P. 2001, A\&A, 370, 194

Massey, P., Armandroff, T. E., Pyke, R., Patel, K., \& Wilson, C. D. 1995, AJ, 110,2715

Mateo, M. 1998, ARA\&A, 36, 435

Meynet, G., \& Maeder, A. 2002, A\&A, 390, 561

Nomoto, K., Iwamoto, K., Nakasato, N., et al. 1997, NuPhA, 621, 467

Peimbert, M. 1978, Planetary Nebulae, , ed. Y. Terzian, IAU Symp. 76, 215

Peimbert, M. 1985, Rev. Mex. A\&A, 10, 125
Peimbert, A., Peimbert, M., \& Ruiz, M. T. 2005, ApJ, 634, 1056 Peña, M., Richer, M. G., \& Stasinska, G. 2007, A\&A, 466, 75

Porter, R. L., Ferland, G. J., \& MacAdam, K. B. 2007, ApJ, 657, 327

Richer, M. G., \& McCall, M. 1995, ApJ, 445, 642

Richer, M. G., \& McCall, M. 2007, ApJ, 658, 328

Seaton, M. 1979, MNRAS, 185, 5

Shaw, R. A., \& Dufour, R. J. 1995, PASP, 107, 896

Stasińska, G., Richer, M., \& McCall, M. 1998, A\&A, 336, 667

Storey, P. J., \& Hummer, D. G. 1995, MNRAS, 272, 41

Venn, K. A., Lennon, D. J., Kaufer, A., et al. 2001, ApJ, 625, 754

Wang, W., \& Liu, X.-W., 2008, MNRAS, 389, L33

Woosley, S. E., \& Weaver, T. A. 1995, ApJS, 101, 181 